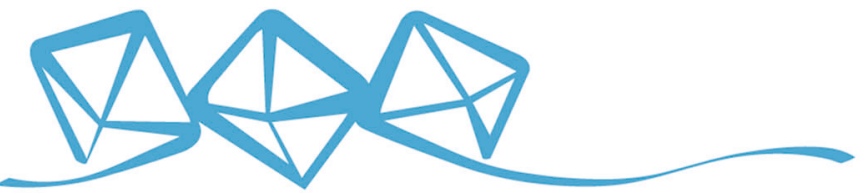 COMMUNICATIONS MATERIALS
}

\section{Piezomagnetic switching and complex phase equilibria in uranium dioxide}

\author{
Daniel J. Antonio', Joel T. Weiss², Katherine S. Shanks'2, Jacob P. C. Ruff ${ }^{3}$, Marcelo Jaime (1) ${ }^{4,9}$, Andres Saul (1) ${ }^{5}$, \\ Thomas Swinburne (1) ${ }^{5}$, Myron Salamon ${ }^{4}$, Keshav Shrestha ${ }^{1,10}$, Barbara Lavina ${ }^{6}$, Daniel Koury6, \\ Sol M. Gruner (1) 2,3, David A. Andersson7 ${ }^{7}$ Christopher R. Stanek7 , Tomasz Durakiewicz ${ }^{1}$, James L. Smith ${ }^{7}$, \\ Zahirul Islam ${ }^{8} \&$ Krzysztof Gofryk (1) ${ }^{1 凶}$
}

Actinide materials exhibit strong spin-lattice coupling and electronic correlations, and are predicted to host new emerging ground states. One example is piezomagnetism and magneto-elastic memory effect in the antiferromagnetic Mott-Hubbard insulator uranium dioxide, though its microscopic nature is under debate. Here, we report X-ray diffraction studies of oriented uranium dioxide crystals under strong pulsed magnetic fields. In the antiferromagnetic state a [888] Bragg diffraction peak follows the bulk magnetostriction that expands under magnetic fields. Upon reversal of the field the expansion turns to contraction, before the [888] peak follows the switching effect and piezomagnetic 'butterfly' behaviour, characteristic of two structures connected by time reversal symmetry. An unexpected splitting of the [888] peak is observed, indicating the simultaneous presence of timereversed domains of the 3-k structure and a complex magnetic-field-induced evolution of the microstructure. These findings open the door for a microscopic understanding of the piezomagnetism and magnetic coupling across strong magneto-elastic interactions.

\footnotetext{
${ }^{1}$ Idaho National Laboratory, Idaho Falls, ID, USA. ${ }^{2}$ Laboratory of Atomic and Solid State Physics, Cornell University, Ithaca, NY, USA. ${ }^{3}$ CHESS, Cornell University, Ithaca, NY, USA. ${ }^{4}$ National High Magnetic Field Laboratory, Los Alamos, NM, USA. ${ }^{5}$ Aix-Marseille University, CINaM-CNRS UMR 7325 Campus de Luminy, Marseille, France. ${ }^{6}$ University of Nevada, Las Vegas, Las Vegas, NV, USA. ${ }^{7}$ Los Alamos National Laboratory, Los Alamos, NM, USA. ${ }^{8}$ Advanced Photon Source, Argonne National Laboratory, Lemont, IL, USA. ${ }^{9}$ Present address: Electrical Quantum Metrology Dep., National Metrology Institute,

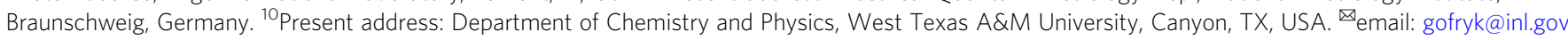


S trong coupling between magnetism and lattice vibrations can lead to many emerging phenomena, as have been shown in unconventional superconductors, heavy-fermions, multiferroics, and other new functional materials ${ }^{1-5}$. Due to strong spin-orbit coupling, correlated $5 f$-electron spin systems represent a perfect platform to scrutinize the phenomena related to spin-phonon interactions, especially when interacting with other degrees of freedom such as multipolar ordering or Jahn-Teller interactions ${ }^{6,7}$. An excellent example is uranium dioxide $\left(\mathrm{UO}_{2}\right)$. This antiferromagnetic Mott-Hubbard insulator ${ }^{8}$ is the main nuclear fuel and the most studied actinide material to date $^{9}$. Its correlated ground state is characterized by a competition among non-collinear magnetic dipoles, electric quadrupoles, and dynamic Jahn-Teller distortions ${ }^{10-12}$. Recently, it has been shown that, due to the magnetic symmetry of the non-collinear 3$\boldsymbol{k}$ antiferromagnetic order (shown in the inset of Fig. 1a) and strong magneto-elastic coupling, $\mathrm{UO}_{2}$ undergoes a trigonal distortion under magnetic field and becomes a piezomagnet with exceptionally large coercive characteristics ${ }^{13}$. In piezomagnetic crystals, a magnetic moment can be induced by the application of physical stress ${ }^{14,15}$. This phenomenon has captured attention in recent years as a mechanism that could be used, in combination with multiferroics and piezoelectrics (especially at the nanoscale), to achieve control of magnetism by electric fields ${ }^{16}$. Piezomagnetism is also utilized in geology where the so-called volcanomagnetic effect is used for monitoring volcanic activities ${ }^{17-19}$. Despite intensive work, the microscopic nature and crystallographic evidence of piezomagnetism are still elusive ${ }^{20,21}$.

Here we show a direct micro-structural probe of piezomagnetism using single-crystal X-ray diffraction. By using a highresolution back-reflection geometry setup (see Supplementary Fig. 4 for details), we were able to resolve small changes in the [888] Bragg peak of $\mathrm{UO}_{2}$ when the magnetic field is applied along the [111] direction, which corresponds to the longitudinal lattice response along the parallel cube diagonal of its crystal lattice. In the paramagnetic state (above $30.5 \mathrm{~K}$ ), the [888] Bragg peak moves toward larger $2 \Theta$ values (see "Methods") with the applied magnetic field, exhibiting negative magnetostriction. When the $\mathrm{UO}_{2}$ crystal is cooled below the magnetic transition temperature (below $30.5 \mathrm{~K}$ ), the application of a magnetic field causes positive magnetostriction in agreement with the measurements of the macroscopic variation of the sample length using a fiber Bragg grating (FBG) technique ${ }^{13}$. When the magnetic field direction is reversed, the sample initially compresses until a critical field is reached and then rapidly expands. The overall behavior resembles the magneto-elastic "butterfly" previously seen with the FBG magnetostriction measurements ${ }^{13}$ and represents piezomagnetic switching between two magnetic structures connected by the time-reversal symmetry. X-rays offer additional insight not available via bulk FBG methods-specifically, we observe a splitting of the [888] peak under the magnetic field. This arises from time-reversed (TR) domains of $3-\boldsymbol{k}$ magnetic structures that have different responses to the applied magnetic fields. To the best of our knowledge, this study represents the first crystallographic observation of piezomagnetism and the switching effect in general, and in a $5 f$-electron spin system in particular.

\section{Results and disscusion}

The thermal expansion and magnetostriction in the paramagnetic state of $\mathrm{UO}_{2}$. The presence of a sudden volume collapse in the unit cell of $\mathrm{UO}_{2}$ at low temperatures has been known for some time 22,23 . The small discontinuity corresponds to the rapid and simultaneous magnetic, electrical, and structural transition at $T_{\mathrm{N}}=30.5 \mathrm{~K}$. Comparing the relative change in the $d$ spacing corresponding to the [888] peak with temperature, which was taken using back-reflection geometry X-ray diffraction, to previously taken dilatometry on single crystal $\mathrm{UO}_{2}$ in the $<111>$ direction $^{13}$ in Fig. 1a, one can see that the diffraction reproduces the expected behavior. The conversion from the angle of the Bragg peak to micro-strain (or p.p.m.) is made using: $\Delta L / L=[\sin$ $\left.\theta_{0} / \sin \theta^{\prime}\right]-1$, where $\theta^{\prime}$ is the Bragg angle of the [888] peak at a given temperature or applied magnetic field; $\theta_{0}$ is its zero-field value. The precision of both techniques is comparable, showing
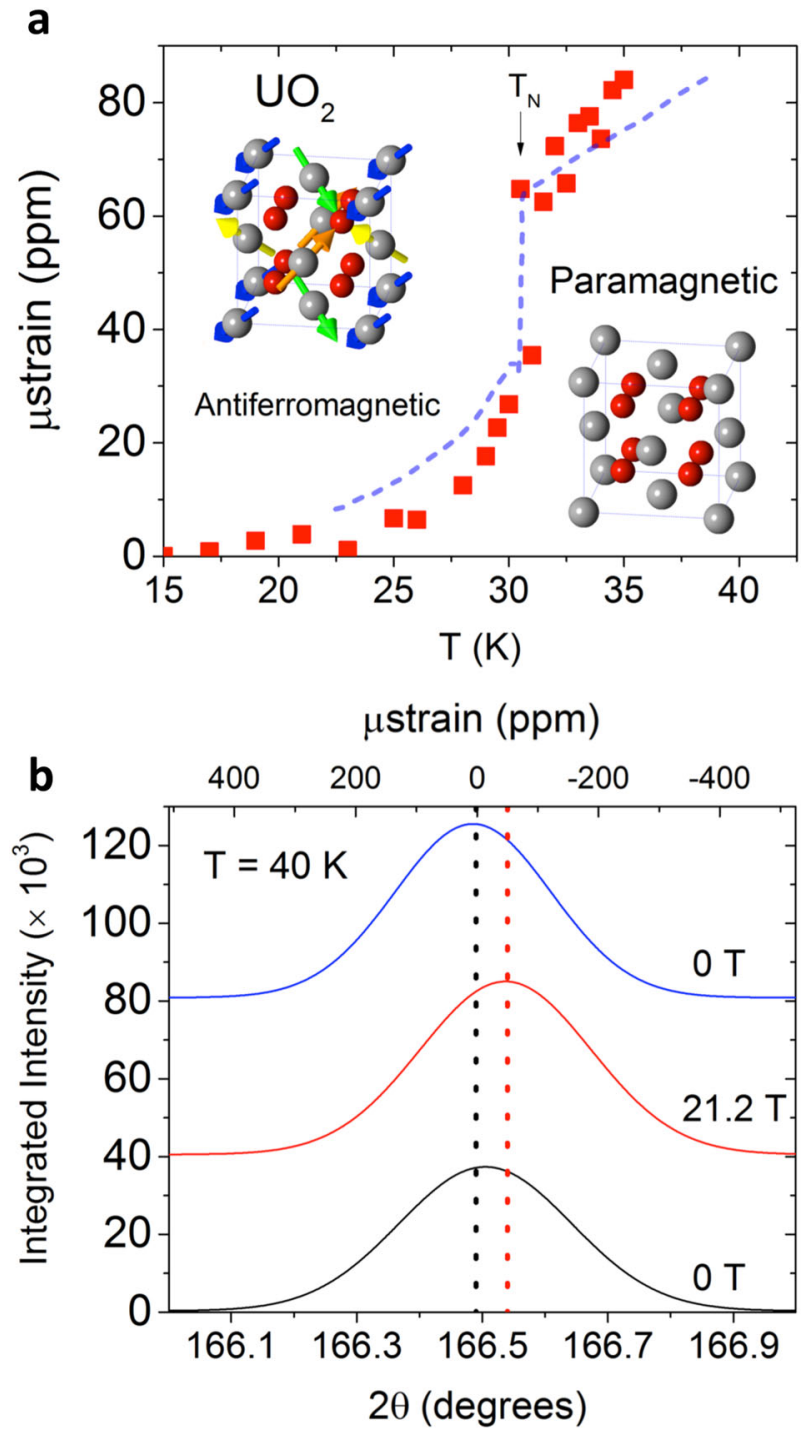

Fig. 1 Thermal expansion and magnetostriction in the paramagnetic state of $\mathbf{U O}_{2}$. a The relative change of the lattice constant in the <111> direction of the $\mathrm{UO}_{2}$ single crystal as a function of temperature, measured by dilatometry of the bulk crystal (dashed line) and X-ray diffraction of the [888] peak (red squares). The $X$-ray data were obtained by single crystal diffraction in high-angle back-reflection geometry using $15.85 \mathrm{keV} \mathrm{X}$-rays. An abrupt collapse in unit cell volume can be seen at $T_{N}=30.8 \mathrm{~K}$, marked by an arrow. Insets show the structure of the $\mathrm{UO}_{2}$ cubic unit cell above $T_{\mathrm{N}}$ (paramagnetic phase) and below $T_{\mathrm{N}}$ (ordered phase). b The magnetic response of the [888] peak in the paramagnetic phase $(T=40 \mathrm{~K})$ to an applied pulsed magnetic field in the <111> direction. The initial state at $0 \mathrm{~T}$ (black line) shifts to higher $2 \theta$ at maximum applied field of $21.2 \mathrm{~T}$ (red line), then back to the same initial value after the pulse (blue line). The shift in $2 \theta$ of the peak corresponds to a small 50 p.p.m. strain contraction in the $<111>$ direction, consistent with bulk single crystal magnetostriction measurements ${ }^{2}$. 
approximately a -30 p.p.m. strain change in the length along the $<111>$ direction at $30.5 \mathrm{~K}$. This shows that the volume collapse at $T_{\mathrm{N}}$ can be observed as the material goes from a paramagnetic state to the ordered $3-\boldsymbol{k}$ antiferromagnetic state. When subjected to a pulsed magnetic field in the paramagnetic state $(T=40 \mathrm{~K})$, the diffraction shows a small contraction of the unit cell along the $<111>$ direction of about 50 p.p.m. at the maximum field of 21.2 $\mathrm{T}$, as seen in Fig. 1b, which also matches the FBG magnetostriction measurements ${ }^{13}$. The structure then reversibly changes back after returning to zero field.

The magnetostriction in the antiferromagnetic state of $\mathrm{UO}_{2}$. When repeating the measurements in the magnetically ordered state, a different and unexpected response occurs. Represented schematically in Fig. 2a (not to scale), FBG magnetostriction measurements in a pulsed magnetic field below $T_{\mathrm{N}}$ revealed that the bulk crystal expanded in the $<111>$ direction, the reverse of the non-magnetic state. While measuring diffraction in the backreflection geometry, the sample was cooled to $15 \mathrm{~K}$, then subjected to a pulsed field in the $\langle 111\rangle$ direction. Under the application of the magnetic field, the [888] peak splits and reconverges as the applied field rises and falls, reversibly returning to its original state after the pulse (see Supplementary Fig. 7 for more details). This surprising result contrasts with the single peak seen in the non-magnetic state. Moreover, the split peaks shift in opposite directions, i.e. higher and lower scattering angles $(2 \Theta)$, indicating simultaneous contraction and expansion of the same crystal lattice. This can only occur if X-ray diffraction originates in two types of physically distinct, three-dimensionally ordered regions of the sample with a robust local symmetry producing fully coherent Bragg peaks. Furthermore, one of the domains (red peak) is much more sensitive to the applied magnetic field, i.e. reaching 700 p.p.m. strain in $20 \mathrm{~T}$, than all the other peaks measured. Remarkably, the two regions respond with opposite sign just as one would expect from two magnetic domains related by time reversal in a magnetic material. This is a very unusual observation, hard to explain in the context of the fcc crystal structure of $\mathrm{UO}_{2}$ yet a natural consequence in a piezomagnetic system. We, hence, believe that the splitting arises from TR domains of the 3-k magnetic structure that have opposite responses to the applied magnetic fields. The integrated intensities of the peaks along $2 \theta$ shown in Fig. $2 \mathrm{~b}$ reveal that there is a larger volume fraction of the sample that corresponds to expansion (blue peak), and a smaller component that corresponds to contraction (red peak). The peak positions of these two separate components plotted in Fig. 2c show that, though the contracting component has a lower intensity, the absolute value of its corresponding strain is larger. The peak positions in the rising and falling field overlap symmetrically as well, showing no hysteresis. Repeatedly applying the pulsed field in the same field direction produces the same result. As can be seen from the figure, the magnetostriction obtained here, governed by the blue peak, agrees well with the FBG pulsed field magnetostriction measurements (black line) ${ }^{13}$.

When the sample is subsequently exposed to a pulsed magnetic field applied in the direction opposite that of the previously applied field, startlingly different behavior is seen. Represented schematically in Fig. 3a, the FBG magnetostriction measurements show that in this reversed field direction in the magnetic state, the response along the $\langle 111\rangle$ direction is a linear contraction, displaying broken time-reversal symmetry, a characteristic of the piezomagnetic effect ${ }^{13}$. After reaching a certain temperaturedependent critical applied field strength ${ }^{24}$, the sample rapidly returns to the previous expansion response seen in the single direction applied field used in the first part of the experiment,
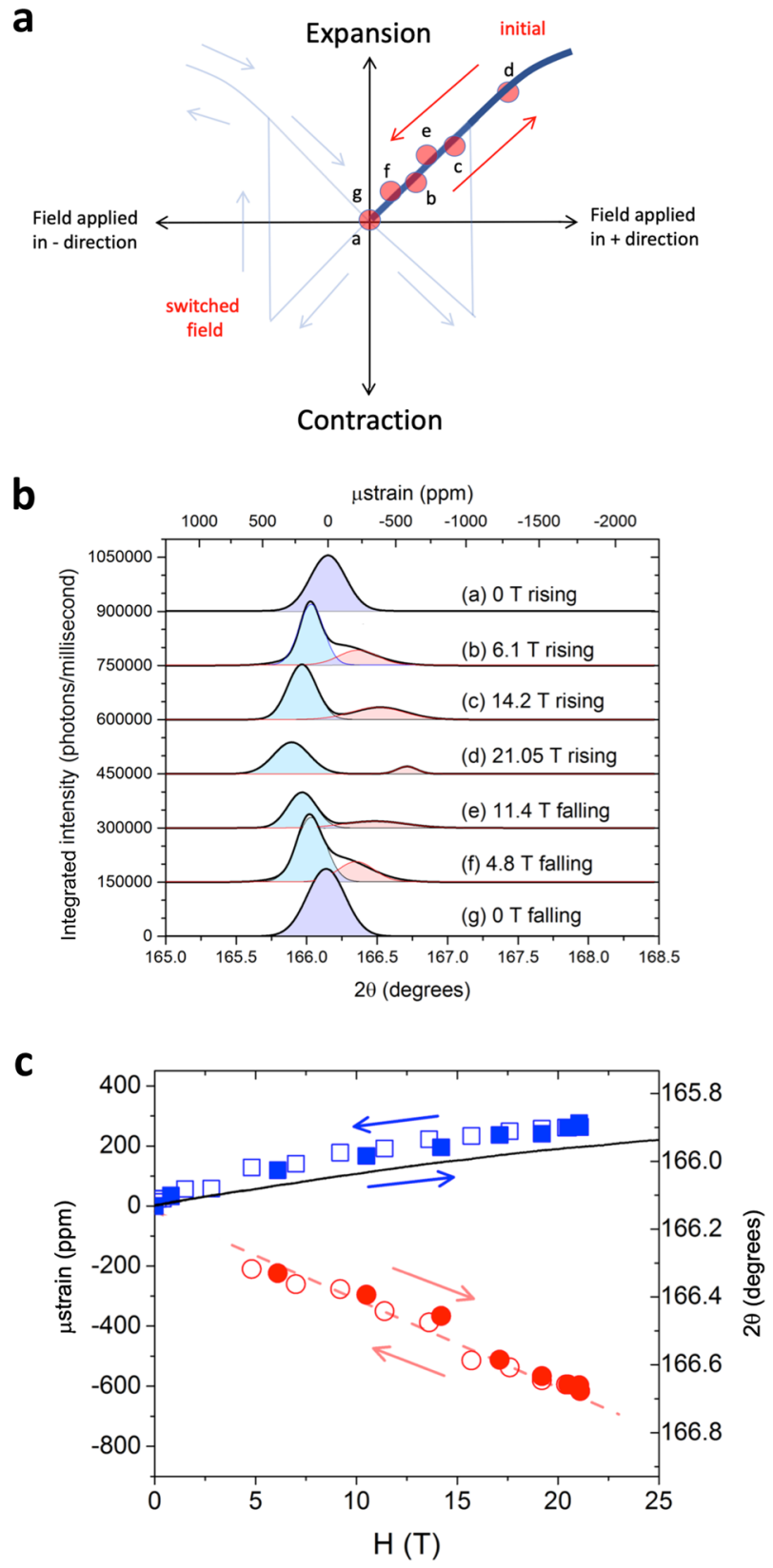

Fig. 2 The magnetostriction in the antiferromagnetic state of $\mathrm{UO}_{2}$-one field direction. a A schematic diagram showing the bulk sample magnetostriction along the <111> direction to a magnetic field applied repeatedly to it in the same direction (bold blue line), showing the sample reversibly expanding. A pale blue pattern shows field dependence of the magnetostriction for positive and negative fields directions. b A waterfall plot of fits to the integrated intensity along $2 \theta$ of selected fields and at $15 \mathrm{~K}$. The peak at lower $2 \theta$ (blue) corresponding to expansion is much larger than the peak at higher $2 \theta$ (red). c The peak positions of the two peaks at $15 \mathrm{~K}$, with filled symbols corresponding to rising field and open symbols to falling field. Red circles represent the smaller peak and blue squares the larger one. The blue peak and its positive magnetostriction can be seen to match previous magnetostriction measurements using the fiber Bragg grating technique (black line), with about a 150 p.p.m. expansion at the maximum $21.1 \mathrm{~T}$ applied field. The red dash line is guide to the eye.

then follows that path back to the same initial state at zero field. Looking at the integrated intensities of the diffracted peaks in Fig. 3b, the behavior of the peaks is very different from the single field direction. As seen there, the overall response is compressive 
a

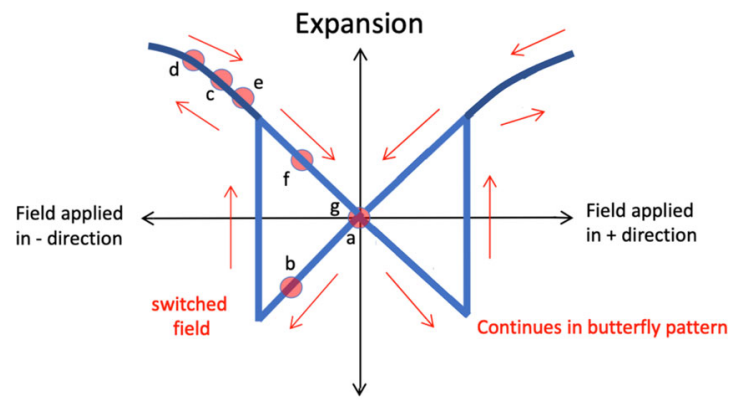

Contraction

b

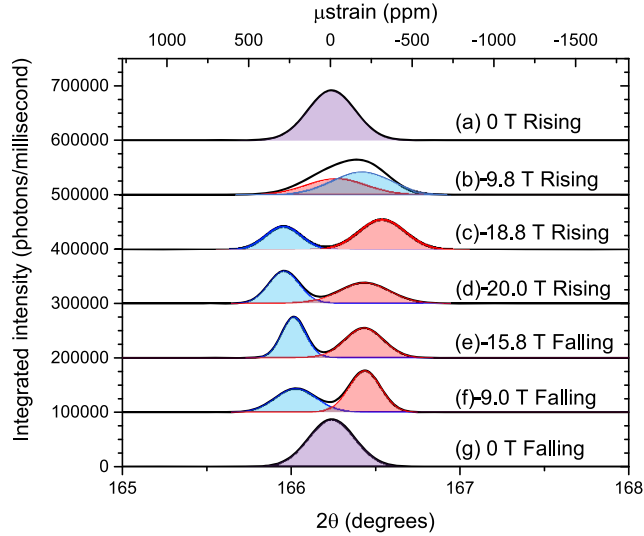

C

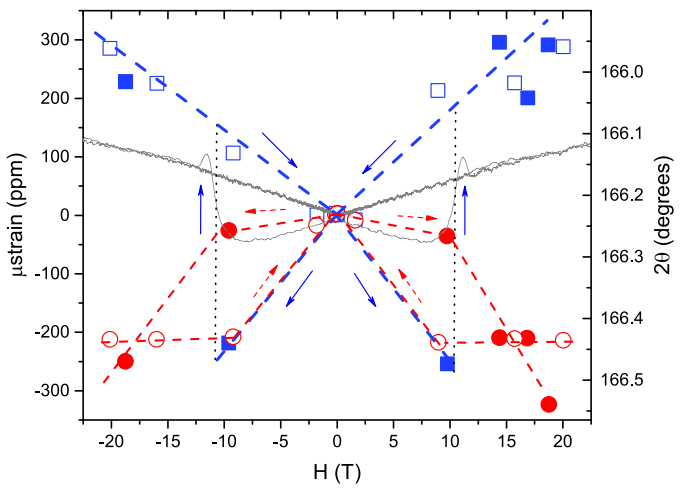

Fig. 3 The magnetostriction in the antiferromagnetic state of $\mathrm{UO}_{2}-$ alternate field directions. a A schematic diagram showing the bulk sample magnetostriction along the $<111>$ direction in a magnetic field applied immediately in the direction opposite to that of the previously applied field (bold blue line). As opposed to Fig. 2a, the sample shows irreversible behavior by instead initially contracting in the $<111>$ direction, but then rapidly switching back to the previous expanding behavior once a threshold field has been surpassed. $\mathbf{b}$ The integrated intensity in $2 \theta$ for selected fields and at temperature, $T=25 \mathrm{~K}$. As opposed to $2 \mathrm{c}$, the higher $2 \theta$ peak is initially larger, but then a change in relative intensities and peak position can be seen. $\mathbf{c} A$ plot of the relative strain calculated from the peak positions in the reversed field state at $25 \mathrm{~K}$. The peak corresponding to the dominant strain behavior as determined by the bulk magnetostriction is the blue squares and the secondary peak corresponding to negative strain is the red circles. Note that the curves for positive fields are similar to the data in Fig. $3 \mathrm{~b}$. Open symbols are used for rising fields and closed for falling fields. The blue solid line is guide to the eye. The solid black line is the reversed field measurements using the FBG technique taken in positive and negative fields at $25 \mathrm{~K}$.

at approximately $-10 \mathrm{~T}$ (see point $b$ in Fig. 3). But then, at increasingly negative field values, the data return abruptly to behavior similar to that seen in Fig. $2 b$, albeit with different relative intensities. The key point is that upon returning from
$-20 \mathrm{~T}$ to zero, the overall compressive strain is not recovered near $-10 \mathrm{~T}$ (point $f$ in Fig. 3). Immediately switching the field direction again shows this same new behavior, resulting in a butterfly-like loop ${ }^{13}$. The peak is clearly seen to split again (first as a broadening of the peak and then as a separate peak), this time in a positive field following immediately after negative pulses at $25 \mathrm{~K}$ (a positive magnetic field part is shown in the Supplementary Fig. 9). The FBG experiments have shown that critical field of about $11 \mathrm{~T}$ is expected at this temperature, as marked by the solid line in Fig. 3b (see Supplementary Fig. 11 for more details and ref. ${ }^{24}$ ). This behavior provides direct evidence that the "butterfly" hysteresis observed in the FBG experiment can therefore be related to piezomagnetic domain evolution revealed by X-rays. Figure $3 \mathrm{c}$ shows the relative positions of the peaks (blue squares and red circles). The lines are visual guides. The higher angle and intensity "blue peak" (point $b$ in Fig. 3) undergoes a switching from contraction to expansion at the critical field of $\sim-11 \mathrm{~T}$. This demonstrates that the sign of the strain in the dominating peak is rapidly reversed in this region so that the blue peak matches the butterfly pattern observed in the magnetostriction taken at the same temperature. If so, then only magnetic domains corresponding to the blue peaks have sufficiently low critical fields to exhibit the rapid reversal of strain behavior expected from the piezomagnetic effect. It has been proposed that, in $\mathrm{UO}_{2}$ crystals subject to pulsed magnetic fields of reversed polarity, the magnetic subsystem switches between two states connected by time reversal ${ }^{13}$. In Fig. $3 c$, this switching phenomenon between the two states is directly observed. It has to be noted that while the coloring of the peaks is not unique, the key point is that upon an initial field reversal only a compressive strain is observed while on the subsequent return to zero field, two peaks are seen. This behavior is the same whether the sample was first trained in positive or negative fields. The two types of behavior apparently cross on initial field reversal, and therefore, that the most sensible labeling of the peaks is as indicated in the text above (see Supplementary Fig. 12 for more details).

In general, the piezomagnetic response, related to $\mathrm{UO}_{2}$ 's fcc, $\mathrm{Pa} \overline{3}$ structure, can be explained using a model Hamiltonian that includes a strong magnetic anisotropy, elastic, Zeeman, Heisenberg exchange, and magnetoelastic contributions to the total energy. This simple model, where the degrees of freedom are the orientation of the magnetic moments of the four $\mathrm{U}$ atoms in the $P a \overline{3}$ unit cell and the shear components of the strain tensor, successfully reproduces the intriguing experimental observations (see Supplementary materials in ref. ${ }^{13}$ for more details regarding the model used). Minimizing the total energy with respect to the elastic shear components allows obtaining their dependence on the applied magnetic field:

$$
\varepsilon_{x y}=\frac{E}{c_{44} a^{3}} M_{\mathrm{st}} H_{z}
$$

with similar expressions for the other components. Here $E=$ $0.280 \mathrm{meVT}^{-1}$ is the strength of the magnetoelastic interaction ${ }^{13}$, $c_{44}=60 \mathrm{GPa}$ and $a=5.47 \AA$ are the experimental shear elastic and lattice constants respectively, and $M_{\mathrm{st}}$ is the staggered magnetization that is different from zero below the Néel temperature. The change of sign of the staggered magnetization, which can be positive or negative depending on which AFM structure connected by time-reversal symmetry is stabilized, allows understanding the observed "butterfly" behavior of the magnetostriction (Fig. 3c).

The phenomenology of peaks splitting under applied magnetic field in $\mathrm{UO}_{2}$ is contrary to the more common case of magnetic detwinning. More typically, the application of magnetic fields to materials containing symmetry-related twin domains will result 
in a free energy difference between the domains, favouring one over the other and driving the material into a single-domain state. Conversely in the case of $\mathrm{UO}_{2}$, at least in the low-field regime, it is clear that the applied magnetic field drives opposing magnetostrictive responses in different TR domains without converting one domain entirely into the other. As seen in Fig. 1, only a single peak, shifted by magnetostriction, appears above the Neel temperature. Also, a single peak is observed above $T_{\mathrm{N}}$ under high pressure (see Supplementary Fig. 2 for more details) and below $T_{\mathrm{N}}$ in the absence of magnetic fields. Because the two-peak structure seen in Figs. 2 and 3 is only observed in the magneticfield-polarized antiferromagnetic state, it is likely that the two magnetic domains consist of distinct TR versions of the $3-\boldsymbol{k}$ structure. The piezomagnetism of $\mathrm{UO}_{2}$ (ref. ${ }^{13}$ ) occurs as the 3-k structure of the ordered phase switches between TR versions under the action of applied magnetic fields. The red circles represent the negative magnetostriction of the $-\mathrm{TR}$ state in positive fields. That this state has not fully converted to the +TR state by a field of $+20 \mathrm{~T}$ indicates that either this TR state is pinned, or that the switching field in this domain is larger than $20 \mathrm{~T}$. The robustness of minority domains to field-conversion may be closely related to the exceptionally hard piezomagnetic response of $\mathrm{UO}_{2}$. Interestingly, the presence of two $180^{\circ}$ antiferromagnetic domains has been observed before in another piezomagnet, $\mathrm{MnF}_{2}$, via polarized neutron tomography ${ }^{25,26}$. Furthermore, the domain configuration was determined to be sensitive to the strain condition of the specimen. It was suggested that for given local stress, the domain configuration (type A and/ or B) depends on the direction of the applied magnetic field, such as the domain type being reversed when the magnetic field points out in the opposite direction ${ }^{25}$. A single domain state is possible if the stress is uniform over the entire crystal. This is a condition difficult to achieve, however, since an increase in the number of domains to attain small-scale variations of stress is prevented by the large wall anisotropy energy. In our experimental configurations, the strain effects in $\mathrm{UO}_{2}$ might play an even bigger role. A potential domain pinning and strain effect imposed by the stycast epoxy (see Fig. 3 in the Supplementary Information) could increase the piezomagnetic switching field so that the preparation of a single TR state becomes increasingly difficult. It has been shown that in other antiferromagnets, such as $\mathrm{NiO}$ crystal $^{27}$, the domain structure has been very sensitive to mechanical stress and magnetic fields, where the twin wall dynamics appear to be limited by a spin-rotation energy loss. We speculate that the sensitivity of the switching field to mechanical stress might be related to the proximity of the mixed antiferromagnetic/ antiferroquadrupolar state of $\mathrm{UO}_{2}$ to the non-magnetic, purely quadrupolar phase ${ }^{28}$. A virtual transition into the quadrupolar state allows for the conversion from one TR state to the other without the need to overcome an anisotropy barrier. In this scenario, internal constraints modify the local distortions that give rise to electric-field gradients and hence reduce the presence of the purely quadrupolar phase. Experiments are underway to explore the sensitivity of the switching field to uniaxial strain in this material. We would like to point out that the 888 peak splitting effect (the presence of two magnetic domains) observed in $\mathrm{UO}_{2}$ is real and does not originate in any way from the superposition of many fields-pulse combinations.

Conclusion. We report the low-temperature single crystal X-ray diffraction studies of $\mathrm{UO}_{2}$ in pulsed magnetic fields. The highresolution single crystal diffraction allows us to study details of subtle unit-cell distortions below and above the structural and magnetic phase transition. We unveil direct microstructural observations of the piezomagnetic and switching effects being a direct consequence of the non-collinear 3-k magnetic order that breaks time-reversal symmetry in a non-trivial way. These results will help in better understanding the strong coupling between magnetic and structural degrees of freedom in this important nuclear material. We also observe the presence of magnetic domains with distinct magnetic-field evolution in the magnetically ordered state of $\mathrm{UO}_{2}$, both when the field is applied repeatedly in a single direction and when the field direction is alternated. We argue that the origin of this behavior is related to the presence of the distinct TR versions of the 3-k structure. This behavior, especially the role of quadrupoles on the piezomagnetic properties, should be investigated in the future to further explore the details of the field-induced broken symmetries.

\section{Methods}

It is worth noting that the quality of the data obtained in the experiment was only possible by bringing together a combination of unique factors. Through the use of an exceptional quality single-crystal sample (evidenced in very narrow diffraction peaks, see Supplementary Fig. 1), high-intensity synchrotron radiation, high, pulsed magnetic fields, and a fast prototype compound area detector, we were able to achieve high precision in our measurements during the short time frame of a pulsed fields (using one of the two choices of $\sim 7$ and $10 \mathrm{~ms}$ in total duration [startto-finish] currently available). The single crystal of $\mathrm{UO}_{2}$ that was used in these studies is from the same batch as used in previous measurements (refs. ${ }^{5}$ and ${ }^{13}$ ). The crystal was aligned and cut into a plate approximately $500 \times 500 \times 200 \mu \mathrm{m}$, with the [111] crystal face normal to the plane. This allows us to study the longitudal magnetostriction of the $\mathrm{UO}_{2}$ crystal along the desired $<111>$ crystolagraphic direction. The experiment was carried out in a dual cryostat single solenoid pulsedmagnet system described in ref. ${ }^{29}$, in beamline 6 ID-C of the Advanced Photon Source at Argonne National Laboratory.

The detector used was a compound-type Mixed-Mode Pixel Array Detector (MM$\mathrm{PAD}$ ) with a silicon sensor ${ }^{30,31}$. This allowed for high dynamic range (up to $2 \times 10^{7} \mathrm{x}-$ rays/pixel/frame at $15.85 \mathrm{keV}$ ), low background single photon counting, and a fast frame rate so that multiple images at different rising and falling field strengths during pulses could be obtained. The angular $2 \Theta$ range of the beam, where $2 \Theta$ is the angle between the incident and scattered rays, was calibrated on the area detector using the [888] peak of a silicon reference crystal, mounted at the center of sample theta rotation of the cryostat. The collection window for each frame was about $140 \mu \mathrm{s}$ at a frame rate of $1 \mathrm{kHz}$, and the detector array was $\mathrm{ca} .4 \mathrm{~cm} \times 6 \mathrm{~cm}$. For temperature scans in zero field, the sample was stabilized at various temperatures and then rotated through the Bragg condition at several hundred discrete sample theta positions as frames were taken at each position, then the frame intensities were added to obtain the full [888] peak profile. The center of mass in sample $\theta$ and Bragg peak position $2 \theta$ of the profile were found and converted to the change in d-spacing to find the change in unit cell length along the $<111>$ direction. A similar process was used for measurements in the applied field, with fewer discrete sample $\theta$ rotation positions, limited by the long duty cycle of the magnet for each individual pulse, as elaborated on in the supplementary material (Supplementary Figs. 4-8).

The capacitor bank used in these experiments provided a maximum field of about $21 \mathrm{~T}$ and a pulse width of $\sim 7 \mathrm{~ms}$, as shown in Supplementary Fig. 5, with the ability to internally reverse the direction of the supplied current to the magnet coil. The reversed field part of the experiment was carried out in a similar manner, but after a first pulse in the positive direction (which was then discarded), successive pulses were taken in alternating directions along the $<111>$ direction of the sample crystal. The resulting reversed-field direction frames were then analyzed the same way as the single direction pulses (Fig. $3 b$ and c).

\section{Data availability}

The data that support the findings of this study are available from the corresponding author upon request.

Received: 1 July 2020; Accepted: 14 December 2020; Published online: 05 February 2021

\section{References}

1. Pines, D. Emergent behavior in strongly correlated electron systems. Rep. Prog. Phys. 79, 9 (2016).

2. Sarrao, J. L. et al. Plutonium-based superconductivity with a transition temperature above 18K. Nature 420, 297-299 (2002).

3. Willers, T. et al. Correlation between ground state and orbital anisotropy in heavy fermion materials. Proc. Natl Acad. Sci. USA 112, 2384-2388 (2015).

4. Fleet, L. Multiferroics: in rare form. Nat. Phys. 13, 926 (2017).

5. Gofryk, K. et al. Anisotropic thermal conductivity in uranium dioxide. Nat. Commun. 5, 4551 (2014). 
6. Santini, P. et al. Multipolar interactions in f-electron systems: the paradigm of actinide dioxides. Rev. Mod. Phys. 81, 807 (2009).

7. Moore, K. T. \& van der Laan, G. Nature of the $5 \mathrm{f}$-states in actinide metals. Rev. Mod. Phys. 81, 235 (2009).

8. Gilbertson, S. M. et al. Ultrafast photoemission spectroscopy of the uranium dioxide $\mathrm{UO}_{2}$ Mott insulator: evidence for a robust energy gap structure. Phys. Rev. Lett. 112, 087402 (2014).

9. Murty, K. L. \& Charit, I. An Introduction to Nuclear Matgerials: Fundamentals and Applications (Wiley, 2013)

10. Prokhorov, A. S. \& Rudashevskii, E. G. Magnetoelastic interactions and the single-domain antiferromagnetic state in cobalt fluoride. Kratk. Soobshch. Fiz. 11, 3-6 (1975)

11. Carretta, S., Santini, P., Caciuffo, R. \& Amoretti, G. Quadrupolar waves in uranium dioxide. Phys. Rev. Lett. 105, 167201 (2010).

12. Caciuffo, R. et al. Multipolar, magnetic, and vibrational lattice dynamics in the low-temperature phase of uranium dioxide. Phys. Rev. B. 84, 104409 (2011).

13. Jaime, M. et al. Piezomagnetism and magnetoelastic memory in uranium dioxide. Nat. Commun. 8, 99 (2017).

14. Dzialoshinskii, I. E. The problem of piezomagnetism. Sov. Phys. JETP 6, 621 (1958).

15. Borovik-Romanov, A. S. Piezomagnetism in the antiferromagnetic fluorides of cobalt and manganese. Sov. Phys. JETP 11, 786-793 (1960).

16. He, Q. et al. Electrically controllable spontaneous magnetism in nanoscale mixed phase multiferroics. Nat. Commun. 2, 225 (2011).

17. Carmichael, R. S. Depth calculation of piezomagnetic effect for earthquake prediction. Earth Planet. Sci. Lett. 36, 309-316 (1977).

18. Okubo, Ayako \& Kanda, Wataru Numerical simulation of piezomagnetic changes associated with hydrothermal pressurization. Geophys. J. Int. 181, 1343-1361 (2010).

19. Henyey, T. L., Pike, S. J. \& Palmer, D. F. On the Measurement of Stress Sensitivity of NRM Using a Cryogenic Magnetometer. In: (eds Fuller, M., Johnston, M. J. S. \& Yukutake, T.). Tectonomagnetics and Local Geomagnetic Field Variations. Advances in Earth and Planetary Sciences, vol 5. Springer, Dordrecht. https://doi.org/10.1007/978-94-010-9825-0_15 (1979).

20. Boldrin, D. et al. Giant piezomagnetism in $\mathrm{Mn}_{3} \mathrm{NiN}$. ACS Appl. Mater. Interfaces 10, 18863-18868 (2018).

21. Tarakanov, V. V. Some remarks on the problem of piezomagnetism. Phys. B 284-288, 1213-1214 (2000).

22. Brandt, O. G. \& Walker, C. T. Temperature dependence of elastic constants and thermal expansion for $\mathrm{UO}_{2}$. Phys. Rev. Lett. 18, 1 (1967)

23. White, G. K. \& Sheard, F. W. The thermal expansion at low temperatures of $\mathrm{UO}_{2}$ and $\mathrm{UO}_{2} / \mathrm{ThO}_{2}$. J. Low Temp. Phys. 14, 445 (1974).

24. Jaime, M., Gofryk, K. \& Bauer, E. D. Magnetoelastics of high field phenomena in antiferromagnets $\mathrm{UO}_{2}$ and CeRhIn ${ }_{5}$. IEEE Xplore https://doi.org/10.1109/ MEGAGAUSS.2018.8722665 (2019).

25. Baruchel, J., Schlenker, M. \& Barbara, B. 180 deg Antiferromagnetic domains in $\mathrm{MnFe}_{2}$ by neutron topography. J. Magn. Magn. Mater. 15, 1520 (1980).

26. Baruchel, J. et al. Piezomagnetism and domains in $\mathrm{MnFe}_{2}$. J. Phys. Colloq. 49, C8-1895-C8-1896 (1988).

27. Slack, G. A. Crystalography and domain walls in antiferromagnetic $\mathrm{NiO}$ crystals. J. Appl. Phys. 31, 1571 (1960).

28. Giannozzi, P. \& Erdös, P. Theoretical analysis of the 3-k magnetic structure and distortion of uranium dioxide. J. Mag. Mag. Mater. 67, 75-87 (1987)

29. Islam, Z. et al. A single-solenoid pulsed-magnet system for single-crystal scattering studies. Rev. Sci. Instrum. 83, 035101 (2012).

30. Tate, M. W. et al. A medium-format, mixed-mode pixel array detector for kilohertz x-ray imaging. J. Phys. Conf. Ser. 425, 062004 (2013).

31. Schuette, D. R. "A Mixed Analog and Digital Pixel Array Detector for Synchrotron X-ray Imaging." PhD thesis, Cornell Univ. (2008).

\section{Acknowledgements}

Work by D.A., K.S. and K.G. was supported by the DOE's Early Career Research Program under the project "Actinide materials under extreme conditions". This research used resources of the Advanced Photon Source, a U.S. Department of Energy (DOE) Office of Science User Facility operated for the DOE Office of Science by Argonne National Laboratory under Contract No. DE-AC02-06CH11357. High-field pulsed magnet and a choke coil were installed at the Advanced Photon Source through a partnership with International Collaboration Center at the Institute for Materials Research (ICC-IMR) and Global Institute for Materials Research Tohoku (GIMRT) at Tohoku University. Detector research in S.G.'s laboratory is supported by DOE grant DE-SC0017631. J.P.C. R.'s research at CHESS/CHEXS was supported by the National Science Foundation under awards DMR-1332208 and DMR-1829070. Work at the NHMFL was supported by the National Science Foundation through Cooperative Agreement No. DMR-1644779, the State of Florida, and the U.S. DOE Office of Basic Energy Science Project No. "Science at 100 T."

\section{Author contributions}

K.G. proposed the experimental studies and D.J.A., Z.I. and K.G. designed the research D.J.A., Z.I., J.T.W., K.S.S., J.P.C.R., M.J., B.L., K.S. and K.G. performed the experiments T.D., J.L.S., D.A.A. and C.R.S. provided the $\mathrm{UO}_{2}$ single-crystal samples and S.M.G. provided the MM-PAD detector. D.K. helped in the sample preparation. A.S., T.S., M.J., K.G. and M.S. discussed and developed the theoretical description. All authors helped prepare various pieces of the experimental apparatus, discussed and interpreted the results, and contributed to the preparation and writing of the manuscript.

\section{Competing interests}

The authors declare no competing interests.

\section{Additional information}

Supplementary information The online version contains supplementary material available at https://doi.org/10.1038/s43246-021-00121-6.

Correspondence and requests for materials should be addressed to K.G.

Peer review information Primary handling editor: Aldo Isidori.

Reprints and permission information is available at http://www.nature.com/reprints

Publisher's note Springer Nature remains neutral with regard to jurisdictional claims in published maps and institutional affiliations.

(c) (i) Open Access This article is licensed under a Creative Commons Attribution 4.0 International License, which permits use, sharing, adaptation, distribution and reproduction in any medium or format, as long as you give appropriate credit to the original author(s) and the source, provide a link to the Creative Commons license, and indicate if changes were made. The images or other third party material in this article are included in the article's Creative Commons license, unless indicated otherwise in a credit line to the material. If material is not included in the article's Creative Commons license and your intended use is not permitted by statutory regulation or exceeds the permitted use, you will need to obtain permission directly from the copyright holder. To view a copy of this license, visit http://creativecommons.org/ licenses/by/4.0/.

This is a U.S. government work and not under copyright protection in the U.S.; foreign copyright protection may apply 2021 